\title{
Quantum graph model for rovibrational states of protonated methane
}

\author{
J. I. Rawlinson 1 \\ Department of Applied Mathematics and Theoretical Physics, \\ University of Cambridge, \\ Wilberforce Road, Cambridge CB3 OWA, U.K.
}

\begin{abstract}
We extend the quantum graph model for the protonated methane $\left(\mathrm{CH}_{5}^{+}\right)$molecular ion, allowing for orientational degrees of freedom. This enables us to compute $J>0$ rovibrational states, and we present our results for $J=0,1,2,3$.
\end{abstract}

\section{Introduction}

In [1] it was proposed that the nuclear motion for the protonated methane $\left(\mathrm{CH}_{5}^{+}\right)$system can be usefully approximated by motion on a quantum graph. The authors computed the energies and symmetry properties of the vibrational states, comparing the results with more sophisticated quantum-chemical calculations. The agreement is remarkable given the simplicity of the quantum graph model, in which the relevant internal degrees of freedom are taken to be only one-dimensional (to be compared with seven-dimensional [2, 3] and even twelve-dimensional calculations [4]). These quantum graph calculations were, however, restricted to $J=0$ states as orientational degrees of freedom were neglected.

In this paper we extend these calculations to the $J>0$ sector, allowing us to explore the full rovibrational spectrum for the quantum graph model. It is here that we see the real advantage of the quantum graph approach: the drastic reduction in the number of degrees of freedom allows us to compute states which have been inaccessible to higher dimensional quantum-chemical calculations. We illustrate this by computing the full low-energy spectrum for $J=3$ rovibrational states.

\section{Quantum graph model}

Recall the 120-vertex graph $\Gamma$ (see Figure 1) introduced in [1] . Each point on the graph represents a possible molecular shape for $\mathrm{CH}_{5}^{+}$, with the vertices corresponding to the 120 symmetry-equivalent energy minima on the potential energy surface (PES) and the edges representing low-energy paths between them. The minima have a $C_{s}$ point-group symmetry and can be thought of as a $\mathrm{H}_{2}$ unit sitting on top of a $\mathrm{CH}_{3}^{+}$tripod. Each is connected to three other minima, with two different kinds of paths occuring, indicated by the red and blue edges. The blue edges correspond to an internal rotations of the $H_{2}$ relative to the tripod (this motion takes the configuration through a $C_{s}$-symmetric saddle point). The red edges correspond to a flip motion which exhanges a pair of protons between the $\mathrm{H}_{2}$ and $\mathrm{CH}_{3}^{+}$units (taking the configuration through a $C_{2 v}$-symmetric saddle point). These paths are illustrated in Figure 2.

We assume that, even at low energies, the molecule is not rigid but is able to explore all of this graph by changing its shape. The molecule also has rotational degrees of freedom. So the space $\mathcal{C}$ of all possible configurations of the molecule can be thought of as the product space of the graph $\Gamma$ with the space of possible orientations $S O(3): \mathcal{C} \cong \Gamma \times S O(3)$. Our strategy is to map the (very complex) quantum dynamics of $\mathrm{CH}_{5}^{+}$onto the motion of a particle confined to $\mathcal{C}$.

\footnotetext{
1 email: jir25@damtp.cam.ac.uk
} 


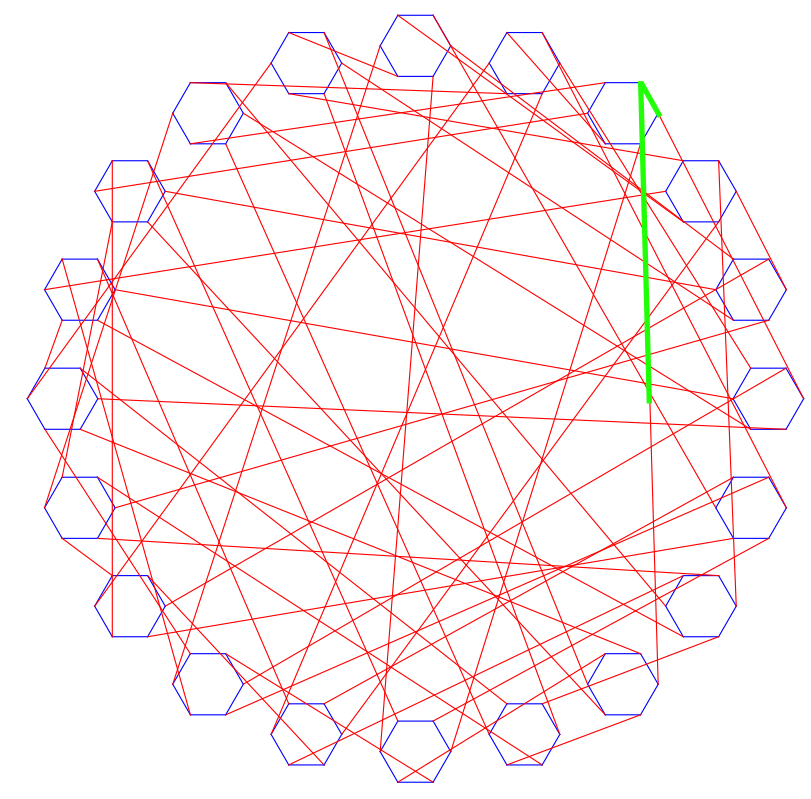

Figure 1: Quantum graph.

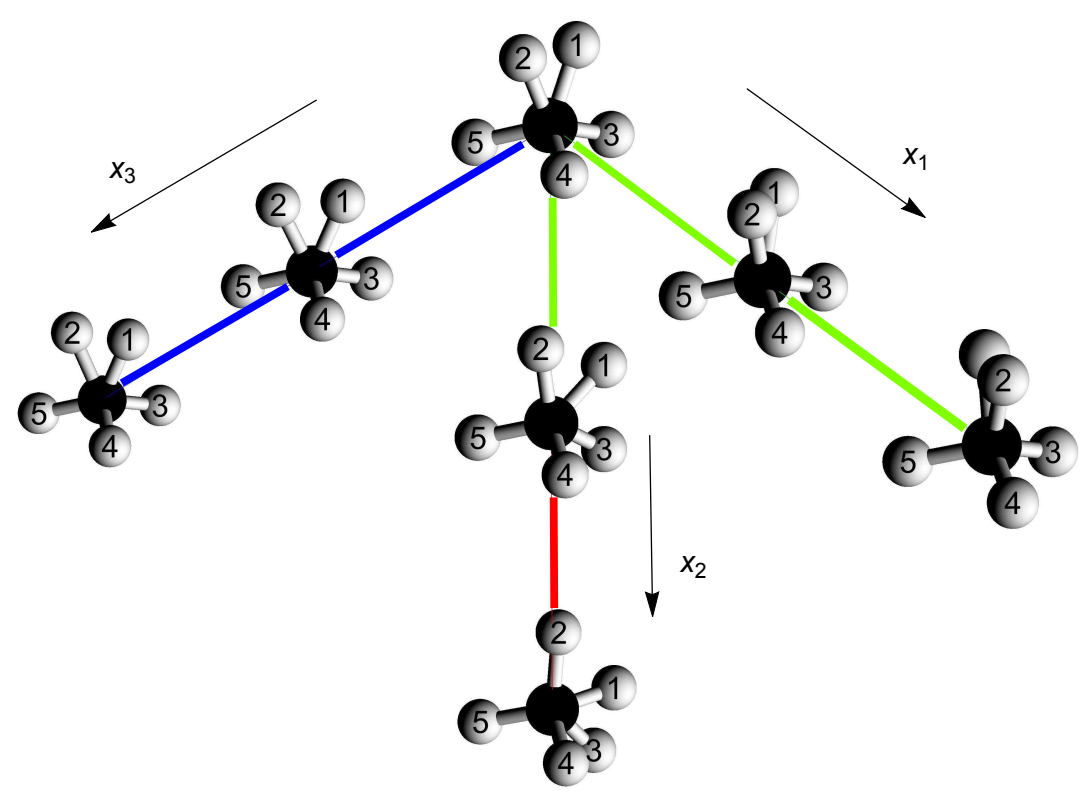

Figure 2: Low-energy paths between minima. 


\section{Motion on $\mathcal{C}$ and symmetries}

The motion of a particle confined to $\mathcal{C}$ is already a drastic simplification compared to the full quantum dynamics of $\mathrm{CH}_{5}^{+}$. However, by exploiting symmetries, we can make the problem even simpler. The Molecular Symmetry (MS) group of $\mathrm{CH}_{5}^{+}$is $S_{5}$, generated by permutations of the 5 protons. Each permutation $\pi \in S_{5}$ acts on $\mathcal{C}$, taking configurations at a given point $p$ on the graph $\Gamma$ and mapping them to configurations at a new point $\pi(p)$ on the graph. In fact, by acting with elements of $S_{5}$ we can generate the entire graph from only two edges, or even one edge and one half-edge. An example of a choice is highlighted in green in Figure 1. We will refer to this green part of $\mathcal{C}$ as the fundamental domain for $S_{5}$, and the vertex where the two green edges meet as $V$. Note that $S_{5}$ is a symmetry of $\mathcal{C}$ and so the quantum states can be classified by irreducible representations (irreps) of $S_{5}$. Working within a particular irrep, the wavefunctions on $\mathcal{C}$ must transform in a definite way under the action of $S_{5}$ and this allows us to deduce the value of the wavefunction on all of $\mathcal{C}$ so long as we know the value of the wavefunction on the fundamental domain. So in fact we only need to determine the wavefunction on the fundamental domain (once an irrep has been chosen), not on all of $\mathcal{C}$ : the rest is determined by symmetry.

\section{Defining the problem on the fundamental domain}

We need to write down an appropriate Hamiltonian on each edge. This should involve a kinetic energy contribution and a potential. In order to make the model as simple as possible we will set the potential to zero. In general, one expects the kinetic energy to involve contributions from both vibrational motion (motion along the graph) and rotational motion as well as so-called rovibrational cross terms. But there is quite a lot of freedom in which coordinates we choose and so we can exploit this freedom to eliminate the cross terms: essentially, we want vibrational motions to be orthogonal to rotational motions.

Start off by picking a coordinate $x^{\prime}$ along the edge of the graph. We will use Euler angles for the orientational degrees of freedom, so altogether we have coordinates $\left(x^{\prime}, \alpha, \beta, \gamma\right)$. The Euler angles $(\alpha, \beta, \gamma)$ tell us (in the usual way) the rotation relating the body-fixed frame of the molecule to a space-fixed frame. But we must still specify a choice of body-fixed frame for each shape along the edge. It is clear that we can make this choice, as we go along the edge, in such a way that compensates for any angular momentum generated by the vibrational motion and thus eliminates any cross terms. So we may assume that in the coordinates $\left(x^{\prime}, \alpha, \beta, \gamma\right)$ the kinetic energy operator has only a purely vibrational contribution and a purely rotational contribution. Now we can further transform the coordinate $x^{\prime} \rightarrow x\left(x^{\prime}\right)$ so as to make the

vibrational kinetic operator simply $-\frac{1}{2} \frac{d^{2}}{d x^{2}}$ (this relies on the fact that the vibration is only one-dimensional). As for the rotational kinetic energy, we assume that the moments of inertia do not vary much and so we use the approximate kinetic energy operator $\frac{1}{2 I} \hat{\mathbf{J}}^{2}$ where $\hat{\mathbf{J}}$ is the generator of body-fixed rotations and $I$ is a constant moment of inertia. Thus we have

$$
\mathcal{H}=-\frac{1}{2} \frac{d^{2}}{d x^{2}}+\frac{1}{2 I} \hat{\mathbf{J}}^{2}
$$

We are now in a position to set up the problem on the fundamental domain.

A vicinity of the fundamental domain is shown in Figure 2, consisting of the vertex $V$ together with the three edges leaving it. Let $x_{1}, x_{3} \in\left[0, L_{1}\right]$ and $x_{2} \in\left[0, L_{2}\right]$ be coordinates along the edges leaving the vertex, with the (green) fundamental domain corresponding to $x_{1} \in\left[0, L_{1}\right]$ and $x_{2} \in\left[0, \frac{L_{2}}{2}\right]$. Suppose $\Psi^{(T)}$ is a state which transforms in the irrep $T$ of $S_{5}$ (so has degeneracy $\operatorname{dim} T$ ) and that for every $\pi \in S_{5}$ we have the corresponding matrix action on the state

$$
\Psi_{n}^{(T)} \rightarrow \sum_{m=1}^{\operatorname{dim} T} T(\pi)_{n m} \Psi_{m}^{(T)} .
$$

On edge $j \in\{1,2,3\}$ the Hamiltonian is

$$
\mathcal{H}_{j}=-\frac{1}{2} \frac{d^{2}}{d x_{j}^{2}}+\frac{1}{2 I} \hat{\mathbf{J}}^{2}
$$


Recall from standard rigid-body theory that the rotational symmetry implies states are classified by quantum numbers $J$ (total angular momentum) and $M \in\{-J, \ldots,+J\}$ (space-fixed angular momentum projection). So we assume that $\Psi_{n}^{(T)}$ is a $(J, M)$ state. We can expand the wavefunction on edge $j$ in terms of $(J, M)$ symmetric-top eigenfunctions (with body-fixed angular momentum projection $K \in\{-J, \ldots,+J\}$ ) and plane waves:

$$
\sum_{K=-J}^{J}\left(a_{n j K} e^{i k x_{j}}+b_{n j K} e^{i k\left(L_{j}-x_{j}\right)}\right)|J K M\rangle
$$

with corresponding energy eigenvalues $E=\frac{1}{2} k^{2}+\frac{1}{2 I} J(J+1)$.

Now recall that we have $S_{5}$ symmetry: for example, the permutation (12) (543) $\in S_{5}$ maps configurations on edge 1 with $x_{1}=x$ to configurations on edge 3 with $x_{3}=L_{1}-x$. The orientations differ by some rotation

$R=\exp (-i \theta \hat{\mathbf{n}} \cdot \hat{\mathbf{J}})(\theta$ and $\hat{\mathbf{n}}$ are estimated in the appendix). We can therefore deduce the wavefunction on edge 3 from the wavefunction on edge 1 . Explicitly, it is

$$
\sum_{m=1}^{\operatorname{dim} T} \sum_{K^{\prime}=-J}^{J} \sum_{K=-J}^{J} T((12)(543))_{n m} \exp (-i \theta \hat{\mathbf{n}} \cdot \hat{\mathbf{J}})_{K K^{\prime}}\left(a_{m 1 K^{\prime}} e^{i k\left(L_{1}-x_{3}\right)}+b_{m 1 K^{\prime}} e^{i k x_{3}}\right)|J K M\rangle .
$$

Now we impose the quantum graph boundary conditions at the vertex $V$ joining edges 1,2 and 3 . These are continuity of the wavefunction together with current conservation, as explained in [1]. As we have expressed the wavefunction on edge 3 in terms of its values on edges 1 and 2, these boundary conditions give us some new conditions relating just the wavefunctions on edges 1 and 2 which have to be satisfied. For example, continuity of the wavefunction at $V\left(x_{1}=x_{2}=x_{3}=0\right)$ leads to

$$
\begin{aligned}
\sum_{m=1}^{\operatorname{dim} T} \sum_{K^{\prime}=-J}^{J} T((12)(543))_{n m} \exp (-i \theta \hat{\mathbf{n}} \cdot \hat{\mathbf{J}})_{K K^{\prime}}\left(a_{m 1 K^{\prime}} e^{i k L_{1}}+b_{m 1 K^{\prime}}\right) & =\left(a_{n 1 K}+b_{n 1 K} e^{i k L_{1}}\right) \\
& =\left(a_{n 2 K}+b_{n 2 K} e^{i k L_{2}}\right)
\end{aligned}
$$

A similar calculation, considering the permutation (23) (45), gives boundary conditions at the midpoint of edge 2. Thus we end up with a set of linear equations in the variables $a_{n 1 K}, a_{n 2 K}, b_{n 1 K}, b_{n 2 K}$ which, as we see in (6), depend on momentum $k$. These are our quantization conditions, and can be handled numerically.

We should note that there is an additional symmetry present, namely inversion in space, which can be used to classifiy states in addition to $S_{5}$. We have found the parity of our computed states by noting that, on edge 2 , spatial inversion can be realised by the combined action of the permutation $(45) \in S_{5}$ followed by a rotation by $\pi$ about the axis normal to the plane of $C_{s}$ reflection symmetry.

\section{Results and discussion}

We display the lowest-lying rovibrational states in Tables 1-4, listed against reference data from the 7dimensional variational calculation in [2]. We have used the values $L_{1}=61.2 \sqrt{m_{e}} a_{0}$ and $L_{2}=1.0 \sqrt{m_{e}} a_{0}$, following the suggestion in [1], to give the best fit to the $J=0$ data (here $m_{e}$ is the electron mass and $a_{0}$ the Bohr radius). For the moment of inertia we have picked a physically reasonable value $\frac{1}{I}=8 \mathrm{~cm}^{-1}$.

We see that the quantum graph states give a good qualitative fit to the reference data even when we extend to $J>0$, with correct $S_{5}$ irrep and parity assignments along with reasonable energy values. The agreement is remarkable considering the simplicity of the quantum graph model. We expect the model to break down at higher energies, where neglected degrees of freedom become important, but these results demonstrate that the graph model is sufficient to understand many states in the low-energy regime. Our $J=3$ states go beyond those computed in [2], in which full spectra were only calculated for $J \leq 2$. Based on the agreement in the $J \leq 2$ sector, we expect our $J=3$ states to be a reliable description of the states of $\mathrm{CH}_{5}^{+}$in the energy range considered. 


\begin{tabular}{|ccc|ccc|}
\hline Irrep & $E$ & $E_{\text {ref }}$ & Irrep & $E$ & $E_{\text {ref }}$ \\
\hline$A_{1}^{+}$ & 0.0 & 0.0 & $G_{2}^{-}$ & 11.4 & 9.9 \\
$H_{1}^{+}$ & 22.2 & 20.4 & $H_{2}^{-}$ & 39.6 & 41.1 \\
$G_{1}^{+}$ & 44.8 & 49.4 & $I^{-}$ & 49.7 & 58.4 \\
$H_{2}^{+}$ & 50.2 & 59.3 & $H_{1}^{-}$ & 96.0 & 113.7 \\
$I^{+}$ & 95.2 & 112.0 & $G_{2}^{-}$ & 100.9 & 112.7 \\
$H_{1}^{+}$ & 112.4 & 122.0 & $H_{2}^{-}$ & 148.8 & 139.4 \\
\hline
\end{tabular}

Table 1: $J=0$ States for quantum graph model (exactly reproducing the results in [1]). The reference data $E_{\text {ref }}$ is from [2].

\begin{tabular}{|ccc|ccc|}
\hline Irrep & $E$ & $E_{\text {ref }}$ & Irrep & $E$ & $E_{\text {ref }}$ \\
\hline$I^{+}$ & 15.3 & 14.7 & $G_{1}^{-}$ & 11.6 & 11.3 \\
$G_{2}^{+}$ & 25.5 & 23.0 & $H_{2}^{-}$ & 27.3 & 24.9 \\
$H_{2}^{+}$ & 32.5 & 31.9 & $I^{-}$ & 30.9 & 29.7 \\
$I^{+}$ & 43.9 & 46.5 & $H_{1}^{-}$ & 38.4 & 40.3 \\
$G_{2}^{+}$ & 51.4 & 57.9 & $A_{1}^{-}$ & 49.9 & 55.4 \\
$H_{1}^{+}$ & 53.4 & 57.1 & $G_{2}^{-}$ & 52.6 & 62.1 \\
$G_{1}^{+}$ & 54.1 & 61.3 & $I^{-}$ & 55.3 & 59.6 \\
$H_{2}^{+}$ & 62.9 & 72.0 & $H_{1}^{-}$ & 57.3 & 67.1 \\
$I^{+}$ & 64.1 & 72.1 & $G_{1}^{-}$ & 58.8 & 62.3 \\
$H_{1}^{+}$ & 92.7 & 115.1 & $H_{2}^{-}$ & 66.6 & 75.5 \\
$G_{2}^{+}$ & 101.4 & 117.1 & $I^{-}$ & 94.9 & 115.0 \\
$H_{2}^{+}$ & 103.1 & 117.2 & $H_{1}^{-}$ & 96.8 & 115.9 \\
$I^{+}$ & 107.2 & 122.7 & $G_{2}^{-}$ & 107.8 & 122.5 \\
$G_{1}^{+}$ & 112.3 & 126.5 & $H_{2}^{-}$ & 109.9 & 122.6 \\
$A_{2}^{+}$ & 113.5 & 125.8 & $G_{1}^{-}$ & 113.8 & 126.3 \\
$H_{2}^{+}$ & 137.0 & 138.7 & $I^{-}$ & 127.1 & 134.0 \\
$I^{+}$ & 154.2 & 145.2 & $H_{1}^{-}$ & 149.9 & 143.4 \\
\hline
\end{tabular}

Table 2: $J=1$ States for quantum graph model.

\begin{tabular}{|ccc|ccc|ccc|ccc|}
\hline Irrep & $E$ & $E_{\text {ref }}$ & Irrep & $E$ & $E_{\text {ref }}$ & Irrep & $E$ & $E_{\text {ref }}$ & Irrep & $E$ & $E_{\text {ref }}$ \\
\hline$H_{1}^{+}$ & 29.5 & 29.1 & $G_{2}^{+}$ & 88.5 & 98.5 & $H_{2}^{-}$ & 32.1 & 31.6 & $G_{1}^{-}$ & 87.8 & 92.0 \\
$G_{1}^{+}$ & 32.2 & 32.2 & $A_{1}^{+}$ & 90.4 & 92.2 & $I^{-}$ & 34.2 & 33.2 & $A_{2}^{-}$ & 99.7 & 126.2 \\
$H_{2}^{+}$ & 40.7 & 39.5 & $G_{1}^{+}$ & 102.1 & 126.1 & $H_{1}^{-}$ & 36.4 & 36.9 & $G_{1}^{-}$ & 101.6 & 126.8 \\
$H_{1}^{+}$ & 46.3 & 46.7 & $G_{2}^{+}$ & 102.6 & 127.9 & $A_{2}^{-}$ & 50.9 & 45.0 & $H_{2}^{-}$ & 103.4 & 126.3 \\
$G_{2}^{+}$ & 52.7 & 48.5 & $I^{+}$ & 105.7 & 126.5 & $G_{2}^{-}$ & 52.2 & 50.0 & $I^{-}$ & 108.0 & 128.0 \\
$I^{+}$ & 53.4 & 53.9 & $H_{1}^{+}$ & 111.5 & 129.8 & $I^{-}$ & 53.3 & 54.6 & $H_{2}^{-}$ & 117.6 & 132.9 \\
$H_{2}^{+}$ & 54.8 & 59.3 & $H_{2}^{+}$ & 115.4 & 134.7 & $H_{2}^{-}$ & 55.3 & 56.4 & $G_{2}^{-}$ & 118.2 & 133.3 \\
$G_{1}^{+}$ & 55.8 & 59.2 & $I^{+}$ & 127.2 & 138.8 & $G_{2}^{-}$ & 58.1 & 64.8 & $H_{1}^{-}$ & 121.1 & 137.6 \\
$A_{2}^{+}$ & 62.0 & 73.3 & $H_{2}^{+}$ & 128.7 & 140.9 & $G_{1}^{-}$ & 60.8 & 66.1 & $I^{-}$ & 127.6 & 141.3 \\
$I^{+}$ & 68.5 & 76.5 & $G_{2}^{+}$ & 140.1 & 147.6 & $H_{1}^{-}$ & 67.4 & 73.4 & $A_{1}^{-}$ & 139.9 & 150.6 \\
$H_{2}^{+}$ & 72.5 & 78.9 & $G_{1}^{+}$ & 146.8 & 152.7 & $I^{-}$ & 68.8 & 74.5 & $G_{2}^{-}$ & 148.8 & 152.0 \\
$H_{1}^{+}$ & 74.3 & 82.6 & $H_{1}^{+}$ & 151.3 & 151.6 & $H_{1}^{-}$ & 76.9 & 82.5 & $H_{1}^{-}$ & 152.4 & 155.2 \\
$G_{1}^{+}$ & 74.9 & 78.1 & $I^{+}$ & 154.6 & 154.3 & $H_{2}^{-}$ & 79.8 & 86.7 & $I^{-}$ & 155.1 & 154.8 \\
$I^{+}$ & 82.5 & 88.5 & $G_{1}^{+}$ & 158.0 & 157.0 & $I^{-}$ & 84.0 & 94.4 & $G_{1}^{-}$ & 157.8 & 152.4 \\
$H_{1}^{+}$ & 86.8 & 95.0 & $A_{1}^{+}$ & 161.8 & 148.4 & $G_{2}^{-}$ & 87.7 & 96.4 & $H_{2}^{-}$ & 171.1 & 162.3 \\
\hline
\end{tabular}

Table 3: $J=2$ States for quantum graph model. 


\begin{tabular}{|cc|cc|cc|cc|}
\hline Irrep & $E$ & Irrep & $E$ & Irrep & $E$ & Irrep & $E$ \\
\hline$I^{+}$ & 56.1 & $G_{1}^{+}$ & 113.1 & $H_{1}^{-}$ & 54.6 & $A_{2}^{-}$ & 115.9 \\
$H_{1}^{+}$ & 59.0 & $H_{1}^{+}$ & 115.3 & $G_{1}^{-}$ & 56.4 & $I^{-}$ & 115.9 \\
$G_{2}^{+}$ & 61.6 & $G_{2}^{+}$ & 118.6 & $I^{-}$ & 59.2 & $H_{2}^{-}$ & 117.7 \\
$H_{2}^{+}$ & 61.9 & $I^{+}$ & 119.8 & $G_{2}^{-}$ & 66.9 & $I^{-}$ & 117.8 \\
$I^{+}$ & 63.0 & $H_{1}^{+}$ & 120.2 & $H_{1}^{-}$ & 67.2 & $G_{1}^{-}$ & 118.7 \\
$G_{1}^{+}$ & 68.1 & $A_{1}^{+}$ & 121.1 & $A_{1}^{-}$ & 68.0 & $H_{2}^{-}$ & 122.8 \\
$H_{2}^{+}$ & 78.4 & $I^{+}$ & 122.1 & $I^{-}$ & 68.7 & $G_{2}^{-}$ & 124.4 \\
$G_{2}^{+}$ & 79.3 & $H_{2}^{+}$ & 125.6 & $H_{2}^{-}$ & 74.6 & $H_{1}^{-}$ & 124.8 \\
$I^{+}$ & 80.4 & $H_{1}^{+}$ & 131.9 & $H_{1}^{-}$ & 79.3 & $G_{1}^{-}$ & 125.4 \\
$A_{2}^{+}$ & 82.0 & $G_{2}^{+}$ & 140.0 & $G_{1}^{-}$ & 79.7 & $I^{-}$ & 135.7 \\
$I^{+}$ & 83.3 & $H_{2}^{+}$ & 143.3 & $H_{2}^{-}$ & 81.5 & $H_{2}^{-}$ & 140.6 \\
$G_{1}^{+}$ & 88.1 & $I^{+}$ & 143.9 & $G_{2}^{-}$ & 81.6 & $H_{1}^{-}$ & 152.5 \\
$G_{2}^{+}$ & 89.6 & $I^{+}$ & 151.4 & $G_{2}^{-}$ & 90.0 & $H_{2}^{-}$ & 154.3 \\
$H_{1}^{+}$ & 90.0 & $G_{1}^{+}$ & 156.4 & $I^{-}$ & 92.8 & $G_{1}^{-}$ & 156.6 \\
$H_{2}^{+}$ & 92.3 & $G_{1}^{+}$ & 171.4 & $I^{-}$ & 97.5 & $G_{2}^{-}$ & 157.7 \\
$H_{1}^{+}$ & 99.5 & $H_{1}^{+}$ & 171.4 & $H_{1}^{-}$ & 98.0 & $A_{1}^{-}$ & 170.8 \\
$I^{+}$ & 103.8 & $G_{2}^{+}$ & 173.6 & $G_{1}^{-}$ & 99.7 & $G_{1}^{-}$ & 176.0 \\
$G_{2}^{+}$ & 111.7 & $H_{2}^{+}$ & 174.5 & $H_{2}^{-}$ & 106.0 & $H_{1}^{-}$ & 176.8 \\
$H_{2}^{+}$ & 112.7 & $H_{1}^{+}$ & 185.1 & $H_{1}^{-}$ & 112.3 & & \\
\hline
\end{tabular}

Table 4: $J=3$ States for quantum graph model.

\section{Appendix}

\section{Estimating $R$}

A reasonable approximation is to take the positions of the protons to be on the surface of a sphere (centred on the Carbon nucleus) as illustrated below. We take the bond angles to be those which give the closest match of the proton positions to $a b$ initio values: the polar angle of the $H_{2}$ unit (in radians) is taken to be 0.42 while the polar angle of the other three protons is taken to be 1.89 .

Consider configurations along edge 1 . Our choice of body-fixed axes $(x, y, z)$ are indicated in the picture: notice that as the $\mathrm{H}_{2}$ unit rotates relative to the $\mathrm{CH}_{3}$ tripod, the entire molecule also rotates at a rate such that the total angular momentum vanishes. The vanishing of the angular momentum ensures that, for this choice of body-fixed axes, there are no kinetic rotation-vibration cross-terms. The rates of rotation are related by the ratio of the moments of inertia, $I_{1}$ and $I_{2}$, of the $H_{2}$ and of the whole molecule. In particular, by the point that the $H_{2}$ unit has rotated a full $\frac{2 \pi}{6}$ with respect to the $\mathrm{CH}_{3}$ tripod, the molecule as a whole will have rotated in the opposite sense by $\Delta \theta=\left(\frac{I_{1}}{I_{2}}\right)\left(\frac{2 \pi}{6}\right)$. Then the rotation relating the configurations at the two endpoints of edge 1 is a rotation by $\frac{2 \pi}{3}+\Delta \theta \approx 2.21$ about the body-fixed $z$-axis, and so we take

$$
R=\exp (-i \theta \hat{\mathbf{n}} \cdot \hat{\mathbf{J}})
$$

with

$$
\theta \approx 2.21, \hat{\mathbf{n}}=\left(\begin{array}{c}
0 \\
0 \\
-1
\end{array}\right)
$$

in our boundary conditions. Edge 2 is treated similarly. 

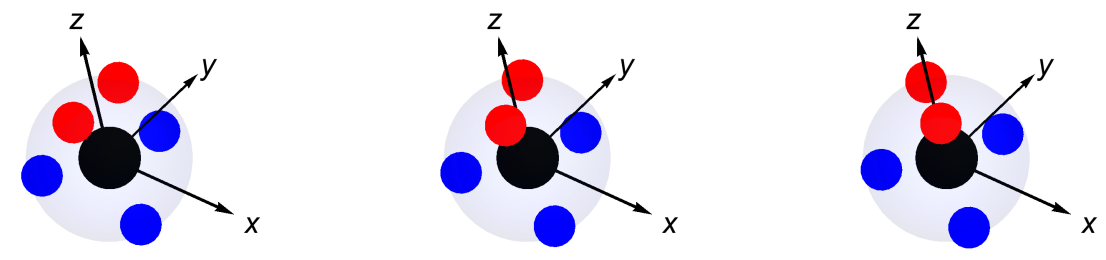

Figure 3: Choice of body-fixed axes.

\section{Acknowledgements}

I am grateful to Csaba Fabri and Attila G. Csaszar for providing Figure 1 and for useful comments. I am supported by an EPSRC studentship. This work has been partially supported by STFC consolidated grant $\mathrm{ST} / \mathrm{P} 000681 / 1$.

\section{References}

[1] C. Fabri and A. G. Csaszar, "Vibrational quantum graphs and their application to the quantum dynamics of $\mathrm{CH}_{5}^{+}$," Phys. Chem. Chem. Phys. 20, 16913 (2018)

[2] X-G. Wang and T. Carrington, "Calculated rotation-bending energy levels of $\mathrm{CH}_{5}^{+}$and a comparison with experiment," J. Chem. Phys. 144, 204304 (2016)

[3] C. Fabri, M. Quack and A. G. Csaszar, "On the use of nonrigid-molecular symmetry in nuclear motion computations employing a discrete variable representation: A case study of the bending energy levels of $\mathrm{CH}_{5}^{+}$," J. Chem. Phys. 147, 134101 (2017)

[4] X-G. Wang and T. Carrington, "Vibrational energy levels of $C H_{5}^{+}$," J. Chem. Phys. 129, 234102 (2008) 